\title{
Comparison Analysis between Route-locking-sectional-releasing and Route-locking-route-releasing Formulations for the Train Platforming Problem in Busy Complex Rail Passenger Stations
}

\author{
Qiongfang Zeng ${ }^{1}$, Yinggui Zhang ${ }^{1 *}$, Dingyou Lei ${ }^{1 *}$ and Li Wang ${ }^{2}$ \\ ${ }^{1}$ School of Traffic and Transportation Engineering, Central South University, \\ Changsha 410075, China \\ ${ }^{2}$ School of Traffic and Transportation, Beijing Jiaotong Univeristy, Beijing \\ 100044, China
}

\begin{abstract}
In this paper, route-locking-route-releasing $(R R)$ interlocking formulation and routelocking-sectional-releasing (RS) interlocking formulation for the train platforming problem (TPP) in busy complex stations are discussed quantitatively. Firstly, considering different operations of various train types conducted in complex passenger stations, a microscopic simulation of train movements in the interlocking area within the station layout is proposed, which aims at calculation of claiming and releasing time instants of station resources in track-circuited formulation for TPP. Then, job shop scheduling models are built on basis of train priorities, and with proposed dispatching rules and a rule-based meta-heuristic algorithm, realistic size problems can be solved in acceptable time. Finally, good quality solutions gained by the RR model and RS model within limited computation time under various dispatching rules are compared. The result shows that good quality solutions original form RS model are statistically better than those from RR model after certain computing time when the track changing strategy is used in complex stations with partially coincident and interconnected routes.
\end{abstract}

Keywords: busy complex passenger stations; train platforming problem; route-lockingsectional-release interlocking; job shop scheduling model

\section{Introduction}

Railway transport, as the green and sustainable means of transportation, is increasing its market share in many countries all around the world. As the train platforming problem (TPP), it refers that trains are needed to be assigned to platforms and tracks reasonably in a railway station, and there is a routing assignment plan specifying for each train within time slots during which the platform/track will be occupied by certain train. The complexity of TPP (also namely train routing problem) in busy complex passenger stations will be added due to the increasingly widespread of high-speed railway and passenger dedicated railway network in China. Thus, such assignment processes in busy complex passenger stations are mainly at the manual processing or at least computeraided artificial processing stage. Train scheduling problem can be categorized into the strategic, tactical and operational levels [1-2], and during the past decades, TPPs have been studied substantially and deeply as discussed by Cacchiani et al. [3]. Also, the train platforming phase, following the timetabling phase, is to find a conflict-free assignment for trains-platforms in a railway station and also corresponding routes in its station layout.

Due to the complexity of railway traffic, most of existing literatures related to Job Shop Scheduling Theory (JSST) and train scheduling focused on macroscopic models for timetabling and routing of railway corridors and networks, in which route nodes or stations between block sections were simplified as special kinds of block sections. For

*Corresponding Authors: ygzhang@csu.edu.cn (Y. Zhang); ding@csu.edu.cn (D. Lei). 
instance, researchers such as Szpigel (1973), Mascis (2002), D'ariano (2007), D'ariano (2008), Mannino (2009) and Corman (2010) viewed trains, block sections and traversing of a block section by a train as jobs, machines and operations respectively, and each station section have one to $N$ parallel tracks and orders of trains are thus considered when arranging trains passing through block sections [4-9]. Implicit representation of route incompatibilities in some large station interlocking areas will become urgent issues when dealing with multiple interactions between inbound and outbound routes. Therefore, developing models with fine granularity of interlocking area is necessary and vital for the practical application of optimal track assignment plans made in advance.

Notably, high frequency of train arrivals or high accuracy requirement of arrival and departure time of trains, especially for the high-speed rail, requires a more detailed microscopic model to explicitly represent the train's movement inside the station layout. In reality, most complex stations are using the route-locking-sectional-releasing interlocking system that is based on the conflicts-free of track-circuits. In recent years, a lot of researchers such as Krasemann (2012), Caimi et al. (2012) and Lusby et al. (2013) proposed microscopic resources (i.e. single block section or single track-circuits) based formulations and heuristics [10-12]. Corman et al. (2009b) compared two formulations to analyze the incompatibility between station routes: one based on sectional-locking and sectional-release of track sections and another based on the route locking and sectionalrelease of aggregated track sections [13]. Although the aggregated formulation with nonsequence-dependent setup times has shown that a good approximation of sectional-release route locking operations may result in small extra buffer times, it is still present some discrepancies with route-locking and sectional-release operations based on track sections in station interlocking area. Also, Caimi et al. (2012) proposed a model predictive control based solution approach for dispatching trains in complex railway stations by assigning predetermined blocking stairways to trains, in which the resulting optimization problem is described as a binary linear programming problem [11]. Moreover, Pellegrini et al. (2014) designed a mixed-integer linear programming formulation for tackling this problem [14]. The route-locking-sectional-release (RS) model is based on the assumption that all track circuits in a block section are occupied at the moment that trains enters at the first trackcircuit of this block and that each track circuit will be released separately when the train rear runs out the track circuit. They compared this model to block or route release models and found that solutions with the route-locking-route-releasing (RR) interlocking system implied statistically significant longer delays suffered by trains than these with the RS interlocking system when considering the possibility of changing train routes. Those researchers have considered track-circuits allowing the modeling of two variants of interlocking systems: a RR interlocking, in which the utilization of a block-section locks all block-sections sharing a track-circuit with it independently on the actual position of the train within the block-section itself; a RS interlocking, in which the utilization of a blocksection locks the block-section sharing with it a not-yet-released track-circuit. Remark that they assumed that the last track-circuit of a block section is usually long enough for hosting the whole train. However, most of the track-circuits in inbound or outbound routes in complex station area are not long enough to contain a train in our experimental analysis and usually trains are not allowed to be stopped at the block section of inbound or outbound routes in the interlocking area within stations except for emergencies.

In this paper, we will focus on developing a track-circuit based model for the TPP in the interlocking area of complex station with various train types and multiple alternative routes and platforms for each train. In order to build a track-circuits based scheduling model, we have to attach importance to claim and release time instants calculation of track-circuits. Although few existing papers such as Sels et al. (2014) considered train speed limits and train length's influence on route claiming and releasing time instants [2], none of existing papers have a clear representation of different operating procedures of various train types specific to complex passenger stations. Thus, we will analyze different 
operations of different train types (e.g. arrival trains, departure trains, turnaround trains, stop through trains, non-stop through trains etc.) conducting in complex stations and further propose a microscopic simulation of train movements in the interlocking area within given station layouts. In the RR interlocking of our version, the utilization of an inbound (outbound) route locks all track-circuits at the moment that home (start) signal gives the cleared aspect to certain train and all track-circuits are released at the same time when the train rear exits the last track-circuit in the route; in the RS interlocking, the utilization of an inbound (outbound) route locks all track-circuits within it once the moment that home (start) signal gives the cleared aspect to certain train and the trackcircuit in the route is released at the moment when the train rear exits the track-circuit.

Furthermore, Törnquist et al. (2007) pointed out that it is also quite common, especially for the rescheduling problem, to use expert systems and priority rules [15]. Those approaches can incorporate the current work process of the dispatchers in various ways by translating tacit knowledge and rules into computerized systematic reasoning. Also, some researchers including Carey et al. (2003) and Carey et al. (2007) developed heuristics analogous to those successfully adopted by station dispatchers using manual methods [16-17]. Although they experimented with different train orders such as random order, chronological order and reverse chronological order when choosing the next train to be considered for scheduling in complex passenger stations, other orders may be ruled out because the train order when choosing the next considering train for scheduling may be nonsense in the station with relatively low density of train arrivals and departures and simple station layout. However, the complexity of the feasibility problem of TPP was studied in detail in Kroon et al. (1997), and he pointed out the feasibility problem is NPcomplete if each train can have three available route-deviation combinations which is the case in our version of TPP [18]. Such NP-hard problems are typically solved via metaheuristic algorithms that can enable to search good quality solutions in a computation time compatible with real-time operations [19]. Since rescheduling in railway networks is time-critical, meta-heuristic approaches are only permitted to run in limited iterations in order to meet the time requirements [20]. The solution based on certain train order (dispatching rule) can be used as an initial solution of the proposed meta-heuristic algorithm. In this paper, we formulate the TPP problem in busy complex stations as a jobshop problem and further design a rule-based meta-heuristic to get a best possible and feasible track assignment plan within limited computation time. Also, with various dispatching rules, good quality solutions with RS interlocking surroundings and RR interlocking surroundings are analyzed in our version of track-circuit based models.

The remainder of this paper is organized as follows. Section 2 defines characteristics and backgrounds of technical operations in busy complex railway passenger stations. This is followed by a detailed simulation of train's movement and functions of station resources' claiming and releasing time instants. Section 3 identifies a train's TPP as the job shop problem and presents both RS interlocking formulation and RR interlocking formulation for the TPP in busy complex passenger stations. Section 4 describes proposed dispatching rules and a rule-based meta-heuristic to solve the above model. Section 5 gives numerical experiments and the comparisons of the results. Section 6 concludes with conclusions and discussions of our further research.

\section{Concepts and Notations}

This section will give a detailed description of the concepts, assumptions and the characteristics and backgrounds of technical operations in busy complex railway passenger stations in our version of TPP. Operation processes of different train types are described, and a more detailed simulation of train movements based on track-circuits will be presented. Further, a detailed calculation method of claiming and releasing time instants of track-circuits and tracks are put forward. 


\subsection{Concepts and Assumptions in Our TPP Version}

To ensure the safety and convenience of operations organization, track utilization in railway passenger stations should abide by the following general rules:

(1) A train can only occupy an arranged track at the same interval, and if certain track has already been reserved by the train, the train can't switch to other tracks in the midway. Also, we assume that each track can accommodate only one train at the same time. We define the occupation time interval of a resource as the time interval, from its claiming time instant to its releasing time instant. In addition, the mapping relationships between any platform and its alternative inbound or outbound routes, and between any route and all the track-circuits, as parts of the route, are both included in the interlocking relationship or chart of a station designed by stations developers and builder.

(2) Each train must have feasible inbound and outbound routes to pass through the station safely. Multiple alternative inbound (outbound) routes can guide the train from entry (exit) point to a track safely, and one of these routes is named as the dominating route and the rest are named as flexible routes. Also, dispatchers often give the highest priority to the dominating route and flexible routes are considered if the dominating route cannot be assigned safely. Mapping relationships between trains and their alternative tracks are dependent on train types, facilities available on the track, and preferences of tracks for different trains, which are dependent on many factors, such as distance from the egress gate of the station and experiences from station dispatchers. Also, mapping relationships between tracks and platforms are determined by the station layout.

(3) The marshalling formation of passenger trains is relatively fixed, and it is unnecessary to break-up and sort the whole passenger train, since carriages may serve for fixed train numbers. The serving work of originating and terminating passenger trains' carriages are usually done in the passenger carriages servicing depot which is also termed as passenger technology station. Hence, apart from motorized carriages, shunting locomotives should be arranged to conduct placing-in and taking-out operations of all the other carriages between the reception-departure yard and passenger technology station However, service work for ORPTs and TEPTs carriages are usually conducted in the passenger carriages servicing depot. After occupying at their assigned tracks, TEPTs alight all their passengers within a standard service time, and the shunting locomotives should be arranged to place TEPTs into the railway passenger technical station. The trains may be decoupled and then coupled in the technical station to make up new ORPTs. Then, the shunting locomotives should be arranged to take ORPTs out of the technical station to their assigned tracks. After boarding all the passengers within the standard service time, ORPTs will start up and depart from the station. ATUPTs terminating at the station can be changed into DTUPTs that will return to its originating station through maintaining carriages in the assigned track or changing the locomotive positions. To better formulate the problem and calculate the track occupation time interval, we can integrate ATUPT and DTUPT that share the same carriages and the same track into one fictive TUPT.

(4) Trains passing through certain complex passenger stations differ in their types, speeds, priorities, preferred routes, tracks and platforms. According to train services and technical operational processes, passenger trains can be classified into originating passenger trains (ORPTs), terminating passenger trains (TEPTs), arrival turnaround passenger trains (ATUPTs), departure turnaround passenger trains (DTUPTs), and through passenger trains (THPTs). Specifically, THPTs can be classified into non-stop through passenger trains (NSTHPTs) and stop through passenger trains (STHPTs) according to whether they will stop at the station or not.

With different train operation processes, passenger trains can also be classified into non-terminating passenger trains (NTEPTs), non-originating passenger trains (NORPTs) and non-stop through passenger trains (NSTHPTs). NTEPTs include STHPTs, TUPTs, and ORPTs, all of which have similar departure operations. The departure time of NTEPT in the timetable is the moment that the train starts to occupy and leaves its assigned track. 
NORPTs include STHPTs, TUPTs and TEPTs, all of which have similar arrival operations. The arrival time of NORPT is the moment that the train stops at its assigned track. NSTHPTs are the trains that run through their assigned inbound routes, tracks and outbound routes at the maximum allowable speed sequentially without any extra stop.

As for the train passing through the station with certain direction, assigned track, assigned inbound and outbound routes, we define the following terms that are related to the claiming and releasing time instants computation. As shown in Figure 1, the first dividing point (entry point) of the route divides the inbound route in the station layout and the last blocking section occupied by the train when it approaches to the station layout. Also, the second dividing point divides the last track-circuit of the inbound route from the track. Moreover, the third one divides the track from the outbound route. Besides, the fourth dividing point (exit point) divides its outbound route from the first blocking section occupied by the train when it leaves the station layout. Obviously, the inbound route is the route between the first dividing point and the second dividing point, while the outbound route is the route between the third dividing point and the fourth dividing point. Moreover, train can receive information such as length of the assigned inbound route and location of the stop marker in the assigned track to calculate the reference braking mode curve and then the driver adjusts travel speed manually until the train stops at the track.

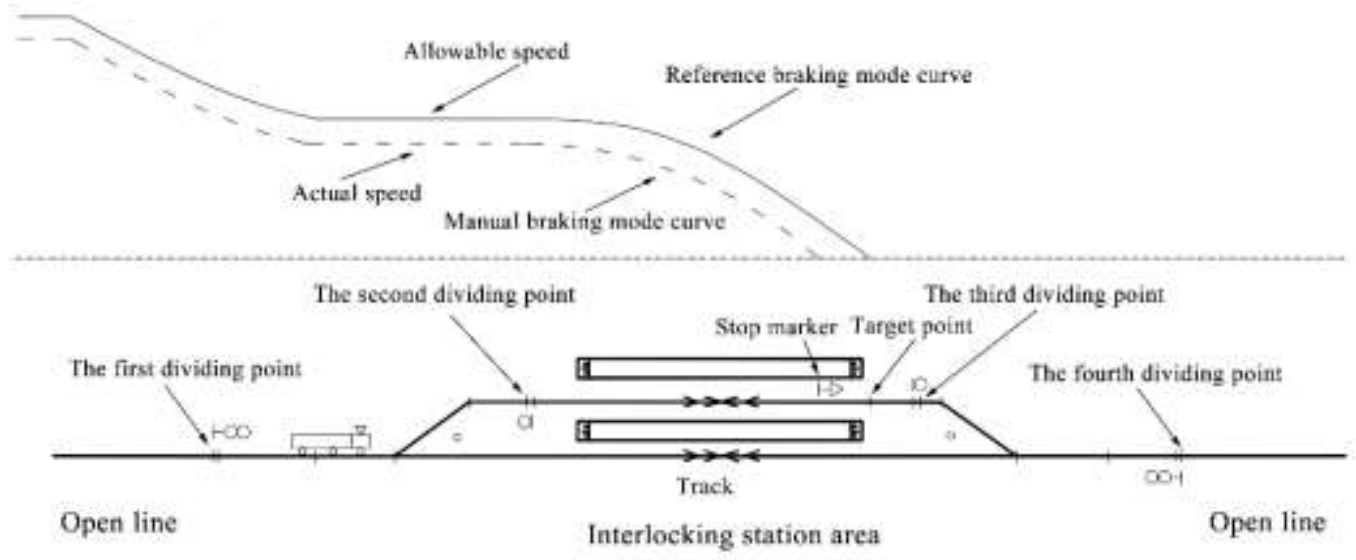

\section{Figure 1. Train Speed Changes and Dividing Points for NORPTs' Arrival Operation along their Routes}

\subsection{Calculation Method for Claiming and Releasing Time Instants of Track-circuits}

This section presents following notations used in the problem formulation described in next few sections. Next, detailed operation processes of various train types and calculation method of claiming and releasing time instant of track-circuits are presented to formulate a track-circuits based scheduling model.

\subsubsection{Notations}

Table 1 and 2 list general subscripts and input parameters, tem-parameters generated during its calculation processing respectively. Also, Table 3 shows decision variables. 
Table 1. Notations for General Subscripts and Input Parameters

\begin{tabular}{|c|c|}
\hline Symbol & Description \\
\hline$x_{i}$ & Arrival time of train $i$ \\
\hline$y_{i}$ & Departure time of train $i$ \\
\hline$\tau_{\text {or }}$ & Standard service time of originating passenger trains \\
\hline$\tau_{t e}$ & Standard service time of terminating passenger trains \\
\hline$J_{i} \in \mathbf{J}$ & $\begin{array}{l}\text { Set of passenger trains indexed by } i . n \text { is the total number of trains passing } \\
\text { through a railway passenger station within a given fixed period. }\end{array}$ \\
\hline $\mathbf{J}_{o r}$ & Set of originating passenger trains \\
\hline $\mathbf{J}_{t e}$ & Set of terminating passenger trains \\
\hline $\mathbf{J}_{t u}$ & Set of fictive turnaround passenger trains \\
\hline $\mathbf{J}_{n s}$ & Set of non-stop through passenger trains \\
\hline $\mathbf{J}_{s t}$ & Set of stop through passenger train station \\
\hline$M_{k} \in \mathbf{M}$ & Set of tracks indexed by $k$, and $K_{m}$ is the total number of tracks \\
\hline$Z_{p} \in \mathbf{P}$ & Set of all platforms indexed by $p$, and $K_{p}$ is the total number of platforms \\
\hline$R_{l} \in \mathbf{R}$ & Set of all routes indexed by $l$, and $K_{r}$ is the total number of routes \\
\hline$S_{q} \in$ Set & Set of all track-circuits indexed by $q$, and $K_{q}$ is their total number \\
\hline$L_{i}$ & Length of train $i$ \\
\hline$L_{l}$ & Length of route $l$ \\
\hline$L_{q}$ & Length of track-circuit $q$ \\
\hline$L^{s g}$ & $\begin{array}{l}\text { Signal visibility distance between the location where the driver can get a } \\
\text { cleared signal and the first dividing point of the train's assigned route. }\end{array}$ \\
\hline$L_{k}$ & Length of track $k$ \\
\hline$L_{k}^{a}$ & $\begin{array}{l}\text { Distance between the location of stop marker in the same direction of the } \\
\text { arrival motion and the second dividing point of the train's assigned route }\end{array}$ \\
\hline$L_{k}^{d}$ & $\begin{array}{l}\text { Distance between the location of stop marker in the same direction of the } \\
\text { arrival motion and the third dividing point of the train's assigned route }\end{array}$ \\
\hline$V_{i}$ & Scheduled speed of train $i$ \\
\hline$V_{l}$ & Maximum allowed speed for NTEPTs or NORPTs in route $l$ \\
\hline$V_{i}^{n s}$ & Maximum allowed speed for NSTHPT $i$ passing through the station \\
\hline$a_{i}$ & Average deceleration of train $i$ during its arrival procedure \\
\hline$d_{i}$ & Average acceleration of train $i$ during its departure procedure \\
\hline$t^{p a}$ & $\begin{array}{l}\text { Reaction and operation time after receiving an arrival signaling aspect } \\
\text { plus the additional time for braking }\end{array}$ \\
\hline$t^{p d}$ & $\begin{array}{l}\text { Reaction and operation time after receiving a starting signaling aspect plus } \\
\text { additional time for starting up }\end{array}$ \\
\hline$\omega_{i}$ & Weight of train $i$ according to its attributes such as types and classes \\
\hline$c_{i p}$ & $\begin{array}{l}\text { Time cost of inconvenience if train } i \text { is allocated to the non-preferred } \\
\text { platform } p\end{array}$ \\
\hline $\mathbf{J}-\mathbf{M}$ & Mapping relationships between trains and tracks \\
\hline M-P & Mapping relationships between tracks and platforms \\
\hline $\mathbf{M}-\mathbf{R}$ & Mapping relationships between tracks and routes \\
\hline $\mathbf{R}-\mathbf{S}-\mathbf{H}$ & Mapping relationships between routes and track-circuits \\
\hline $\mathbf{J}_{t u}^{a}-\mathbf{J}_{t u}^{d}$ & $\begin{array}{l}\text { Mapping relationships between ATUPTs and DTUPTs sharing the same } \\
\text { passenger carriages and the same track }\end{array}$ \\
\hline
\end{tabular}




\begin{tabular}{|c|c|}
\hline$\theta_{i}$ & $\begin{array}{l}\text { Limitation: number of the alternative tracks that are allowed to be claimed } \\
\text { by the train } i\end{array}$ \\
\hline$Q_{i}$ & Set of alternative tracks of train $i$ \\
\hline$Q_{i k}^{a}$ & Set of alternative inbound routes for train $i$ ending at the track $k$ \\
\hline$Q_{i k}^{d}$ & Set of alternative outbound routes for train $i$ starting from the track $k$ \\
\hline$K_{l}$ & Number of the track-circuits that make up route $l$ \\
\hline Set & $\begin{array}{l}\text { Set of track-circuits claimed sequentially by a train when the train runs } \\
\text { along the route } l\left(\operatorname{Set}_{l}=\left\{S_{1}^{l}, S_{2}^{l}, \cdots, S_{h}^{l}, \cdots, S_{K_{l}}^{l}\right\}\right)\end{array}$ \\
\hline$L_{h}^{l}$ & $\begin{array}{l}\text { If the track-circuit } q\left(S_{q} \in \text { Set }\right) \text { is the } h \text {-th track-circuit } S_{h}^{l} \text { in } S e t_{l} \text { claimed } \\
\text { by train } i \text { when it goes through the route } l \text {, then the length of the track- } \\
\text { circuit is denoted by } L_{h}^{l}\left(L_{h}^{l}=L_{q}\right) \text {. }\end{array}$ \\
\hline
\end{tabular}

Table 2. Notations for Temp-parameters Generated while Calculations

\begin{tabular}{cl}
\hline Symbol & Description \\
\hline $\mathbf{J}^{\prime}$ & $\begin{array}{l}\text { Processing sequencing list, } \mathbf{J}^{\prime}=\left\{\boldsymbol{J}_{1}^{\prime}, \ldots, J_{i}^{\prime}, J_{j}^{\prime} \ldots, J_{n}^{\prime}\right\} . \text { For any different } \\
\text { trains } \boldsymbol{J}_{i}^{\prime} \text { and } \boldsymbol{J}_{j}^{\prime}(i \neq j), \boldsymbol{J}_{j}^{\prime} \text { is prior to } \boldsymbol{J}_{i}^{\prime}\end{array}$ \\
$t_{z}^{k}$ & The $z$-th occupation time interval of track $k$ \\
$\Gamma$ & Unassigned train set \\
$S(i, k)$ & Claiming (Start) time instant of track $k\left(k \in Q_{i k}\right)$ occupied by the train $i$ \\
$F(i, k)$ & Releasing (Finish) time instant of track $k\left(k \in Q_{i k}\right)$ occupied by train $i$ \\
$S\left(i, l, o_{i k l}\right)$ & Claiming (Start) time instant of route $l\left(l \in Q_{i k}^{a} \cup Q_{i k}^{d}\right)$ occupied by train $i$ \\
$F\left(i, l, o_{i k l}\right)$ & Releasing (Finish) time instant of route $l$ occupied by train $i$ \\
$S\left(i, l, h, o_{i k l}\right)$ & Claiming time instant of track-circuit $h$ in route $l$ occupied by train $i$ \\
$F\left(i, l, h, o_{i k l}\right)$ & Releasing time instant of track-circuit $h$ in route $l$ occupied by train $i$ \\
$\rho_{k}$ & Total occupation times of track $k$ \\
\hline
\end{tabular}

Table 3. Decision Variables

\begin{tabular}{cl}
\hline Symbol & Description \\
\hline \multirow{2}{*}{$o_{i k}$} & $\begin{array}{l}0-1 \text { binary track variable. If the track } k \text { is claimed by the train } i, \\
\text { then } o_{i k}=1 ; \text { otherwise } o_{i k}=0 .\end{array}$ \\
& $\begin{array}{l}\text { Route variable. If the route } l \text { is claimed by the train } i \text { as an inbound route } \\
\text { ending at the track } k \text {, then } o_{i k l}=1 ; \text { if route } l \text { is claimed by train } i \text { as an }\end{array}$ \\
& outbound route starting from the track $k$, then $o_{i k l}=2 ;$ otherwise $o_{i k l}=0$. \\
& $0-1$ binary track-circuit variable. If track-circuit $q$ is claimed by train $i$ as \\
& part of route $l$ connecting to track $k$, then $o_{i k l q}=1 ;$ otherwise $o_{i k l q}=0$. \\
& $0-1$ binary platform variable. If platform $p$ is occupied by train $i$, \\
& then $o_{i p}=1 ;$ otherwise $o_{i p}=0$. \\
$o_{i p k}$ & $0-1$ binary variable of delayed train. If train $J_{i}$ is not assigned to a \\
$D_{i}$ & complete route through the station, then $D_{i}=1 ;$ otherwise $D_{i}=0$.
\end{tabular}




\subsubsection{Operating Procedure}

In general, stations dispatchers do not shift arrival and departure times in given timetable because they consider time shifting to be as the part of the upper level, and thus claiming and releasing time instants can be derived by subtracting or adding the running time of chosen routes or track-circuits from the arrival and departure time. The claiming and releasing time instants of tracks and track-circuits are determined by the train traction calculation and factors including the length of track-circuits, average acceleration, average deceleration, train scheduled speed, curve radius and gradients of routes and so on. However, some factors such as traction force and motion resistance are hard to acquire in reality. For simplicity, Kroon et al. (1997) and Lu et al. (2004) presented that time instants can be determined using the well-known formulas from the theory of dynamics and assumed that trains have either a uniform motion, or a uniform acceleration or deceleration motion $[18,21]$. Although constraint rates of acceleration and deceleration is slightly unrealistic [12]. It's consistent with what is being done in practice [22-23]. Thus, the departure process can be regarded as combination of processes of the uniform motion and uniform acceleration, while the arrival process can be regarded as combination of processes of the uniform motion and uniform deceleration motion.

Proposition 2.1. If $J_{i} \in \mathbf{J}_{t e}$, then the claiming and releasing time instants of track $k\left(M_{k} \in Q_{i}\right)$ can be expressed by Eq. (1) and (2) respectively; if $J_{i} \in \mathbf{J}_{s t} \cup \mathbf{J}_{t u}$, then the claiming time instant of track $k$ can be expressed by Eq. (1); if $J_{i} \in \mathbf{J}_{t e} \cup \mathbf{J}_{s t} \cup \mathbf{J}_{t u}$, then the claiming time instant of track-circuit $S_{h}^{l}\left(S_{h}^{l} \in S e t_{l}\right)$ of the inbound route $l\left(R_{l} \in Q_{i k}^{a}\right)$ can be expressed by Eq. (3); if $V_{l}^{2} / 2 a_{i} \geq L_{k}^{a}+L_{l}$ or $V_{l}^{2} / 2 a_{i}+L_{i} \geq L_{k}^{a}+L_{l}$, then the releasing time instant of track-circuit $S_{h}^{l}$ can be expressed by Eq. (4). Assuming that the $v\left(v \in\left\{1,2, \cdots, K_{l}\right\}\right)$ th track-circuit $S_{v}^{l} \quad$ satisfies the condition of $\sum_{u=v+1}^{K_{l}} L_{u}^{l} \leq V_{l}^{2} / 2 a_{i}+L_{i}-L_{k}^{a} \leq \sum_{u=v}^{K_{l}} L_{u}^{l} \quad$, if $L_{k}^{a}+L_{l} \geq V_{l}^{2} / 2 a_{i}+L_{i} \geq L_{k}^{a}$ and $h \geq v$, then the releasing time instant of track-circuit $S_{h}^{l}$ can be expressed by Eq. (4); otherwise if $L_{k}^{a}+L_{l} \geq V_{l}^{2} / 2 a_{i}+L_{i} \geq L_{k}^{a}$ and $h \leq v-1$, then the releasing time instant of track-circuit $S_{h}^{l}$ can be expressed by Eq. (5); If $L_{k}^{a} \geq L^{b r 2}+L_{i}$, then releasing time instant of the $h$-th track-circuit is expressed by Eq. (6).

$$
\begin{aligned}
& S(i, k)=x_{i}+\frac{V_{i}-V_{l}}{V_{l}} t^{p a}-\frac{V_{i}}{a_{i}}+\frac{V_{i}^{2}}{2 a_{i} V_{l}}-\frac{L_{k}^{a}+L_{i}+L^{s g}}{V_{l}} \\
& F(i, k)=x_{i}+\tau_{t e} \\
& S\left(i, l, h, o_{i k l}\right)=x_{i}+\frac{V_{i}-V_{l}}{V_{l}} t^{p a}-\frac{V_{i}}{a_{i}}+\frac{V_{i}^{2}}{2 a_{i} V_{l}}-\frac{L_{k}^{a}+L_{i}+L^{s g}}{V_{l}} \\
& F\left(i, l, h, o_{i k l}\right)=x_{i}-\operatorname{sqrt}\left(\frac{2}{a_{i}}\left(L_{k}^{a}-L_{i}+\sum_{u=h+1}^{K_{l}} L_{u}^{l}\right)\right) \\
& F\left(i, l, h, o_{i k l}\right)=x_{i}-\frac{V_{l}}{2 a_{i}}-\frac{1}{V_{l}}\left(L_{i}+L_{k}^{a}+L_{l}-\sum_{u=1}^{h} L_{u}^{l}\right) \\
& F\left(i, l, h, o_{i k l}\right)=x_{i}-\frac{V_{l}}{2 a_{i}}-\frac{1}{V_{l}}\left(L_{k}^{a}-L_{i}+\sum_{u=h+1}^{K_{l}} L_{u}^{l}\right)
\end{aligned}
$$

Proof. The moment when home signal gives the cleared aspect is the claiming time instant of both inbound route and its connected track.

Arrival operation process of train $i$ can be divided into four periods sequentially. The first period is the driver's reaction and operation time after home signal showing cleared aspect. The second period is the first uniform deceleration motion which reduces the train speed from scheduled speed $V_{i}$ to maximum allowed speed $V_{l}$ in the route $l$. The third period is a uniform motion at speed $V_{l}$, and the fourth period is the second uniform 
deceleration motion which reduces the train speed from $V_{l}$ to zero. The first uniform deceleration time gained by reducing the speed from $V_{i}$ to $V_{l}$ can be denoted as $t^{b r 1}=\left(V_{i}-V_{l}\right) / a_{i}$, and its distance is denoted by $L^{b r 1}=\left(V_{i}^{2}-V_{l}^{2}\right) / 2 a_{i}$. The time taken by reducing the speed from $V_{l}$ to zero is denoted by $t^{b r 2}=V_{l} / a_{i}$, and its distance is denoted by $L^{b r 2}=V_{l}^{2} / 2 a_{i}$. Then, the uniform motion time is denoted by $t_{\text {unia }}=\left(L_{l}+L^{s g}-V_{i} t^{p a}+L_{k}^{a}-L^{b r 1}-L^{b r 2}\right) / V_{l}$. Thus, the moment when train stops at track $k$ is denoted as $x_{i}=S(i, k)+t^{p a}+t^{b r 1}+t^{u n i a}+t^{b r 2}$, by which Eq. (1) and Eq. (3) are deduced.

Similarly, the inbound route $l$ of train $i$ is claimed at one time and released by trackcircuits sequentially. The moment that train rear runs out the $h$-th track-circuit in the inbound route $l$ is the releasing time instant of the $h$-th track-circuit. The movement of train rear in the last track-circuit of inbound route can be divided into the following three conditions depending on train length and the second uniform deceleration distance. If $L^{b r 2} \geq L_{k}^{a}+L_{l}$, then movement of train head in inbound route is uniform deceleration motion. Therefore, the releasing time instant of the $h$-th track-circuit can be expressed by Eq. (4). If $L^{b r 2}+L_{i} \geq L_{k}^{a}+L_{l}$, then the movement of train rear in inbound route is uniform deceleration motion. Therefore, the releasing time instant of the $h$-th track-circuit can be expressed by Eq. (4). If $L_{k}^{a}+L_{l} \geq L^{b r 2}+L_{i} \geq L_{k}^{a}$, then the movement of train rear in inbound route is uniform motion and uniform deceleration motion sequentially. Let distance of rear of train' uniform motion in inbound route be denoted by $L_{l}^{c o}$; and let distance of uniform deceleration motion of train rear in inbound route be denoted by $L_{l}^{b r}=L^{b r 2}+L_{i}-L_{k}^{a}$. Assuming that the $v$-th track-circuit $S_{v}^{l}$ satisfy the condition of $\sum_{u=v+1}^{K_{l}} L_{u}^{l} \leq L_{l}^{b r} \leq \sum_{u=v}^{K_{l}} L_{u}^{l}$, then the second uniform deceleration operation takes place in this track-circuit. Therefore, releasing time instant of the track-circuit is denoted by Eq. (4) and (5)according to whether sequence of track-circuit $S_{h}^{l}$ is before or after the trackcircuit $S_{v}^{l}$. If $L_{k}^{a} \geq L^{b r 2}+L_{i}$, then the movement of train rear in inbound route is uniform motion. Thus, the releasing time instant of the $h$-th track-circuit can be gained by Eq. (6).

As for terminating train $i$, arrival time plus standard service time of TEPT $\tau_{t e}$ make the releasing time instant of track $k$, and then Eq. (2) is deduced.

Proposition 2.2. If $\boldsymbol{J}_{i} \in \mathbf{J}_{o r}$, then the claiming and releasing time instants of track $k\left(M_{k} \in Q_{i}\right)$ can be expressed by Eq. (7) and (8) respectively. If $J_{i} \in \mathbf{J}_{s t} \cup \mathbf{J}_{t u}$, then the releasing time instant of track $k\left(M_{k} \in Q_{i}\right)$ can be expressed by Eq. (8). If $J_{i} \in \mathbf{J}_{o r} \cup \mathbf{J}_{s t} \cup \mathbf{J}_{t u}$, then the claiming time instant of outbound route $l\left(R_{l} \in Q_{i k}^{d}\right)$ and the track-circuit $S_{h}^{l}$ in the route can be expressed by Eq. (9) and (10) respectively. If $V_{l}^{2} / 2 d_{i} \geq L_{i}+L_{k}^{d}+L_{l}$, then the releasing time instant of track-circuit $S_{h}^{l}$ can be expressed by Eq. (11). Assuming that the $v$-th track-circuit $S_{v}^{l}$ satisfy the condition of $\sum_{u=v+1}^{K_{l}} L_{u}^{l} \leq V_{l}^{2} / 2 d_{i}-L_{i}-L_{k}^{d} \leq \sum_{u=v}^{K_{l}} L_{u}^{l}$, if $L_{i}+L_{k}^{d} \leq V_{l}^{2} / 2 d_{i} \leq L_{i}+L_{k}^{d}+L_{l}$ and $h \geq v$, then the releasing time instant of track-circuit $S_{h}^{l}$ can be expressed by Eq. (12); otherwise if $L_{i}+L_{k}^{d} \leq V_{l}^{2} / 2 d_{i} \leq L_{i}+L_{k}^{d}+L_{l}$ and $h \leq v-1$, then the releasing time instant of track-circuit $S_{h}^{l}$ can be expressed by Eq. (13). If $V_{l}^{2} / 2 d_{i} \leq L_{i}+L_{k}^{d}$, then the releasing time instant of track-circuit $S_{h}^{l}$ can be expressed by Eq. (12).

$$
S(i, k)=y_{i}-\tau_{o r}
$$




$$
\begin{aligned}
& F(i, k)=\left\{\begin{array}{l}
y_{i}+\operatorname{sqrt}\left(\frac{2}{d_{i}}\left(L_{i}+L_{k}^{d}+L_{l}\right)\right) \frac{V_{l}^{2}}{2 d_{i}}>L_{i}+L_{k}^{d}+L_{l} \\
y_{i}+\frac{V_{l}}{2 d_{i}}+\frac{L_{i}+L_{k}^{d}+L_{l}}{V_{l}} \quad \frac{V_{l}^{2}}{2 d_{i}} \leq L_{i}+L_{k}^{d}+L_{l}
\end{array}\right. \\
& S(i, k)=y_{i}-t^{p d} \\
& S\left(i, l, h, o_{i k l}\right)=y_{i}-t^{p d} \\
& F\left(i, l, h, o_{i k l}\right)=y_{i}+\operatorname{sqrt}\left(\frac{2}{d_{i}}\left(L_{i}+L_{k}^{d}+\sum_{u=h+1}^{K_{l}} L_{u}^{l}\right)\right) \\
& F\left(i, l, h, o_{i k l}\right)=y_{i}+\frac{V_{l}}{2 d_{i}}+\frac{1}{V_{l}}\left(L_{i}+L_{l}-\sum_{u=h+1}^{K_{l}} L_{u}^{l}+L_{k}^{d}\right) \\
& F\left(i, l, h, o_{i k l}\right)=y_{i}+\operatorname{sqrt}\left(\frac{2}{d_{i}}\left(L_{i}+L_{k}^{d}\right)\right)+\operatorname{sqrt}\left(\frac{2}{d_{i}} \sum_{u=1}^{h} L_{u}^{l}\right)
\end{aligned}
$$

Proof. The moment when starting signal gives cleared aspect is the claiming time instant of outbound route. Outbound route $l$ of train $i$ is claimed at one time and released by track-circuits sequentially.

Departure operation process of train $i$ can be divided into four periods sequentially. The first period is driver's reaction and operation time $t^{p d}$ after start signal giving cleared aspect. Therefore, we have Eq. (9) and (10). The second period is the first uniform acceleration motion which increases the train speed from zero to maximum allowed speed $V_{l}$ in route $l$. The third period is a uniform motion at speed $V_{l}$ until train rear runs out of outbound route. The fourth period is the second uniform acceleration motion which increases its speed from $V_{l}$ to $V_{i}$ outside the interlocking station area. The first uniform acceleration time taken by increasing the speed from 0 to $V_{l}$ is denoted by $t^{a c 1}=V_{l} / d_{i}$, and its uniform acceleration distance is denoted by $L^{a c 1}=V_{l}^{2} / 2 d_{i}$.

The movement of train rear in the last track-circuit of the outbound route can be divided into the following three conditions depending on train length, outbound route length and the first uniform acceleration distance. If $L^{a c 1} \geq L_{k}^{d}+L_{l}+L_{i}$, then train rear have uniformly accelerated motion until it runs out of the outbound route. Therefore, time instant of train rear running out of track is the releasing time instant of the track as shown in Eq. (8), and the releasing time instant of the $h$-th track-circuit can be expressed by Eq. (11). If $L_{k}^{d}+L_{l}+L_{i} \geq L^{a c l} \geq L_{k}^{d}+L_{i}$, then the movement of train rear in outbound route is a combination of uniform acceleration motion and uniform motion. After train rear entering outbound route, its uniform acceleration distance in outbound route is denoted by $L_{l}^{a c}=L^{a c 1}-\left(L_{k}^{d}+L_{i}\right)$, and its uniform motion distance in outbound route is denoted by $L_{l}^{c o}=L_{l}+L_{k}^{d}+L_{i}-L^{a c 1}$. Therefore, the moment that train rear runs out of track is the releasing time instant of track as expressed by Eq. (8). Assuming that the $v$-th track-circuit $S_{v}^{l}$ satisfy the condition of $\sum_{u=v+1}^{K_{l}} L_{u}^{l} \leq L_{l}^{a c} \leq \sum_{u=v}^{K_{l}} L_{u}^{l}$, then the first uniform acceleration ends in this track-circuit. Therefore, the releasing time instant of the $h$-th track-circuit is expressed by Eq. (11) and (12) according to whether sequence of track-circuit $S_{h}^{l}$ is before or after the track-circuit $S_{v}^{l}$. If $L^{a c 1}<L_{k}^{d}+L_{i}$, then speed of train rear reaches maximum allowed speed before it enters outbound route, and train rear have a uniform motion in outbound route. Therefore, the releasing time instants of track-circuit in route and track are denoted by Eq. (13) and (8) respectively.

As for originating train $i$, departure time minus standard service time interval of ORPT $\tau_{o r}$ make the claiming time instant of track $k$, and then Eq. (7) is deduced. 
Proposition 2.3. If $J_{i} \in \mathbf{J}_{n s}$, then the claiming and releasing time instants of track $k$ can be expressed by Eq. (14) and (15) respectively; the claiming and releasing time instants of track-circuit $S_{h}^{l}$ of inbound route $l\left(R_{l} \in Q_{i k}^{a}\right)$ can be expressed by Eq. (16) and (17) respectively; and the claiming and releasing time instants of track-circuit $S_{h}^{l}$ of outbound route $l\left(R_{l} \in Q_{i k}^{d}\right)$ can be expressed by Eq. (18) and (19) respectively.

$$
\begin{aligned}
& S(i, k)=x_{i}-t^{p a}-\frac{V_{i}}{a_{i}}-\frac{L^{s g}}{V_{i}}+\frac{V_{i}^{2}}{2 a_{i}}+\frac{V_{i}^{n s}}{2 a_{i}} \\
& F(i, k)=x_{i}+\frac{L_{i}+L_{k}}{V_{i}^{n s}} \\
& S\left(i, l, h, o_{i k l}\right)=x_{i}-t^{p a}-\frac{V_{i}}{a_{i}}-\frac{L^{s g}}{V_{i}}+\frac{V_{i}^{2}}{2 a_{i}}+\frac{V_{i}^{n s}}{2 a_{i}} \\
& F\left(i, l, h, o_{i k l}\right)=x_{i}+\operatorname{sqrt}\left(\frac{1}{V_{i}^{n s}}\left(L_{i}+L_{l}-\sum_{u=h+1}^{K_{l}} L_{u}^{l}\right)\right) \\
& S\left(i, l, h, o_{i k l}\right)=x_{i}-t^{p a}-\frac{V_{i}}{a_{i}}-\frac{L^{s g}}{V_{i}}+\frac{V_{i}^{2}}{2 a_{i}}+\frac{V_{i}^{n s}}{2 a_{i}} \\
& F\left(i, l, h, o_{i k l}\right)=x_{i}+\frac{1}{V_{i}^{n s}}\left(L_{i}+L_{l}+L_{k}-\sum_{u=h+1}^{K_{l}} L_{u}^{l}\right)
\end{aligned}
$$

Proof. The moment that home signal gives cleared aspect is the claiming time instant of inbound route, track and outbound route.

The arrival and departure process of NSTHPTs can be divided into three periods sequentially: the first period is uniform deceleration motion which reduces train speed $V_{i}$ to maximum allowed speed $V_{i}^{n s}$; the second period is uniform motion until train rear runs out of outbound route; the third period is uniform acceleration motion which increases train speed from $V_{i}^{n s}$ to train scheduled speed $V_{i}$. Therefore, the uniform deceleration motion time is denoted by $t^{b r 1}=\frac{V_{i}-V_{i}^{n s}}{a_{i}}$, and its distance is denoted by $L^{b r 1}=\frac{V_{i}^{2}-\left(V_{i}^{n s}\right)^{2}}{2 a_{i}}$. As for NSTHPTs, the arrival and departure time in timetable is the moment that train head reaches stop marker in its assigned track, and it can be denoted by $x_{i}=S(i, k)+\frac{V_{i}}{a_{i}}+\frac{L^{s g}}{V_{i}^{n s}}-\frac{V_{i}^{2}}{2 a_{i}}-\frac{V_{i}^{n s}}{2 a_{i}}$, and then Eq. (14), (16) and (18) are deduced.

The releasing time instant of the last track-circuit in inbound route is expressed by $F\left(i, l, h, o_{i k l}\right)=x_{i}+\frac{L_{i}+L_{k}}{V_{i}^{n s}}$. Therefore, the releasing time instant of the $h$-th track-circuit in inbound route is expressed by Eq. (17), and the releasing time instant of tracks is expressed by Eq. (15). Also, the moment that train rear runs out of the outbound route is denoted by $F\left(i, l, h, o_{i k l}\right)=x_{i}+\frac{L_{i}+L_{l}+L_{k}}{V_{i}^{n s}}$. Therefore, the releasing time instant of the $h$-th track-circuit in outbound route is expressed by Eq. (19).

\section{Model Formulation}

Passenger trains arrive at and depart from the platform according to the given timetable, and the total number of arrivals and departures are fixed within the given time period. If we regard trains as jobs, tracks as paralleled machines, train's transversal of tracks as operations, then processing of trains in complex passenger stations can be formulated as flow shop one with jobs on parallel machines, and TPP can be viewed as a 
job shop scheduling problem with renewable resources (i.e. track-circuits and platforms). With modern scheduling theory, passenger trains set $\mathbf{J}$ is regarded as waiting processing jobs set; arrival time $x_{i}$ of train $i$ is viewed as ready time $r_{i}$ of job; departure time $y_{i}$ is viewed as due date $c_{i}$; and processing time is denoted by $p_{i}=y_{i}-x_{i}$.

The ready time for train $\boldsymbol{J}_{i}$ can be formulated as follows:

$r_{i}= \begin{cases}y_{i}-\tau_{\text {or }} & \text { for ORPT } \\ x_{i} & \text { for others }\end{cases}$

The due date for train $i$ can be formulated as follows:

$$
c_{i}= \begin{cases}x_{i}+\tau_{\text {te }} & \text { for TEPT } \\ x_{i} & \text { for NSTHPT } \\ y_{i} & \text { for others }\end{cases}
$$

Hence, the scheduling model for TPP can be denoted by the 3-field problem classification [24], and expressed as shown in Eq. (22).

$$
P m\left|J_{i}, r_{i}, p_{i}, c_{i}, \omega_{i}, \vartheta_{i}, J-M, J_{t u}^{a}-J_{t u}^{d}, T-P, M-R, R-S-H\right| F
$$

in which the most important objective is to minimize the weighted number of delayed trains as shown in Eq. (23), which has been used in most existing papers on TPP [25].

$$
F=\sum_{i=1}^{n} \omega_{i} D_{i}
$$

\subsection{RS Model: Constraints under RS Interlocking Formulation}

The occupation time interval of train $i$ claiming track-circuit $q$ within the route $l$ which is connected to track $k$, and the occupation time interval of another train $j$ claiming the same track-circuit $q$ in another route $e$, which is connected to track $d$, should be nonoverlapping, otherwise conflicts at the track-circuit $q$ may occur, which is expressed by

$$
\begin{aligned}
& {\left[S\left(i, l, q, o_{i k l}\right), F\left(i, l, q, o_{i k l}\right)\right] \cap\left[S\left(j, e, q, o_{j d e}\right), F\left(J_{j}, e, q, o_{j d e}\right)\right]=\Phi} \\
& i \neq j, \forall o_{i k q}=o_{j d e q}=1, \forall S_{q} \in \text { Set }
\end{aligned}
$$

Also, the occupation time interval of train $i$ claiming track $k$ and the occupation time interval of another train $j$ claiming the same track $k$ should also be non-overlapping, otherwise conflicts at the track $k$ may occur, which can be expressed by Eq. (25).

$$
\begin{aligned}
& {[S(i, k), F(i, k)] \cap[S(j, k), F(j, k)]=\Phi} \\
& i \neq j, \forall o_{i k}=o_{j k}=1, \forall M_{k} \in \mathbf{M}
\end{aligned}
$$

Moreover, the constraint that each train can only claim one track at most is denoted as

$$
\sum_{k=1}^{K_{m}} o_{i k} \leq 1 \quad \forall J_{i} \in \mathbf{J}
$$

and the constraint that each train can only occupy one platform at most is expressed as,

$$
\sum_{p=1}^{K_{p}} o_{i p} \leq 1 \quad \forall J_{i} \in \mathbf{J}
$$

\subsection{RR Model: Constraints under RR Interlocking Formation}

The occupation time interval of train $i$ claiming the route $l$, which is connected to track $k$, and the occupation time interval of another train $j$ claiming the same route $l$, which is connected to track $d$, should be non-overlapping, otherwise conflicts at the route $l$ may occur. Such constraint can be expressed by Eq. (28). 


$$
\begin{aligned}
& {\left[S\left(i, l, o_{i k l}\right), F\left(i, l, o_{i k l}\right)\right] \cap\left[S\left(j, l, o_{j d l}\right), F\left(J_{j}, l, o_{j d l}\right)\right]=\Phi} \\
& i \neq j, \forall o_{i k l}=o_{j d l}=1, \forall R_{l} \in \mathbf{R}
\end{aligned}
$$

Similarly, the occupation time interval of train $i$ claiming track $k$ and the occupation time interval of another train $j$ claiming the same track $k$ should be non-overlapping, which can be denoted as Eq. (25), and the constraints that each train can only claim one track or one platform at most can be expressed by Eq. (26) and Eq. (27) respectively.

\section{Rule-Based Meta-Heuristic}

This section will describe some proposed dispatching rules to solve above job-shop scheduling models, and dispatching rules include basic dispatching rules and composite dispatching rules combining experience from dispatchers and basic dispatching rules. The TPP in railway passenger stations is a composite scheduling problem [25], which can be regarded as synthesis of controllable scheduling, just-in-time scheduling, time window scheduling, renewable resources scheduling and multi-objective scheduling [26-27]. Combining experience from dispatchers and typical optimal rules from classic scheduling problems, various dispatching rules and the rule-based meta-heuristic are both designed.

(1) Basic dispatching rules, e.g. 1) earliest due date first (EDD, $\left.c_{i}\right), 2$ ) first in and first out for train arriving at station (FIFO, $\left.r_{i}\right), 3$ ) shortest processing time first (SPT, $\left.p_{i}\right), 4$ ) weighted SPT first (WSPT, $p_{i} / \omega_{i}$ ), 5) weighted discounted SPT first (WDSPT, $P_{\mathrm{IS}_{-}}$), 6) biggest weight first $\left(\mathrm{BW}, \omega_{i}\right)$ and 7) smallest limitation first (SL, $\theta_{i}$ ).

(2) Composite dispatching rules, e.g., 8) FIFO-EDD, where symbol"-"means both rules are adopted at the same time and the former one is prior to the latter one, 9) EDD-FIFO, and 10) WSPT-FIFO-EDD.

(3) $\theta$ composite rules are these composite dispatching rules with SL, such as, 11) BWSL-FIFO-EDD , and 12) SL-BW-FIFO-EDD .

All sequencing parameters from basic dispatching rules can be determined by its corresponding parameters' value except the parameter $P_{15 \_i}$ for WDSPT, which is calculated by (Pinedo, 2008 [28])

$$
P_{\mathrm{I} 5 \_} i=\left(\omega_{i} \exp \left(-r_{d} p_{i}\right)\right) /\left(1-\exp \left(-r_{d} p_{i}\right)\right)
$$

where $r_{d}$ expresses the discount factor for train $J_{i}$. And the other sequencing parameters for composite and $\theta$ dispatching rules are composition of parameters of basic ones involved in the composite rules.

The solution can be achieved by using scheduling algorithm based on dispatching rules, however, further optimization for being feasible in practice and achieve good solution qualities is also needed. To gain better solutions, we develop a rule-based metaheuristic to achieve the best incumbent solution based on optimization strategies that are refined from station dispatchers' experience. And such heuristic is shown as follows:

Setp1: Initialization. Choose a dispatching rule and get a solution based on certain dispatching rule as incumbent solution.

Step2: Iteration and optimization. A solution improvement strategy can be used to generate new solutions to be assessed in the neighborhood of the best ones tested so far; and a new solution is compared against the incumbent solution. If it is better than the incumbent one, then the incumbent solution will be replaced. A more detailed explanation of rule-based meta-heuristic can be found in the reference of Zhang et al. (2013) [25].

The major solution improvement strategy we used here is a track changing strategy. Usually, there are only one dominating inbound (outbound) route and several default inbound (outbound) routes for certain track in the interlocking chart. Simply adopting another route in the same alternative inbound (outbound) route set linking to the same 
track is not effective enough to avoid conflicts and generate better solutions. Therefore, dispatchers are more focused on changing track assignment, and the assigned inbound or outbound route which is dependent on the given track will be further changed.

\section{Computational Tests and Results}

The above model and algorithm are verified through experimental analysis of TPP in a busy complex passenger station. Here, we reveal some of the experimental results. All the experiments are performed on an IBM Thinkpad SL410 with $2.00 \mathrm{GHz}$ CPU and $1.99 \mathrm{~GB}$ memory, and the algorithm are implemented on a windows XP platform. To prove that train orders when choosing the next considering train for TPPs in complex passenger stations will affect good quality solutions within limited computation time, we will further compare the objective values of RSs based on different rules in Section 5.2.1. Moreover, in Section 5.2.2, good quality solutions generated by the RR model and RS model under different dispatching rules will be further compared.

\subsection{Experimental Setup}

Figure 2 illustrates its schematic layout of Guangzhou East Railway Station as the test station. As shown in Figure 2, there are 4 lines in Shenzhen direction and 2 lines in Guangzhou direction. The station has 14 tracks and 7 platforms. All the platforms can be used in either direction. In reality, there are much more switches and intersections than those as shown in Figure 2 at the interlocking area within the station layout. For example, Figure 3 shows the detail of interconnected routes in one direction of interlocking area. From the interlocking chart of the station, there are 317 inbound and outbound routes and 105 track-circuits in total.

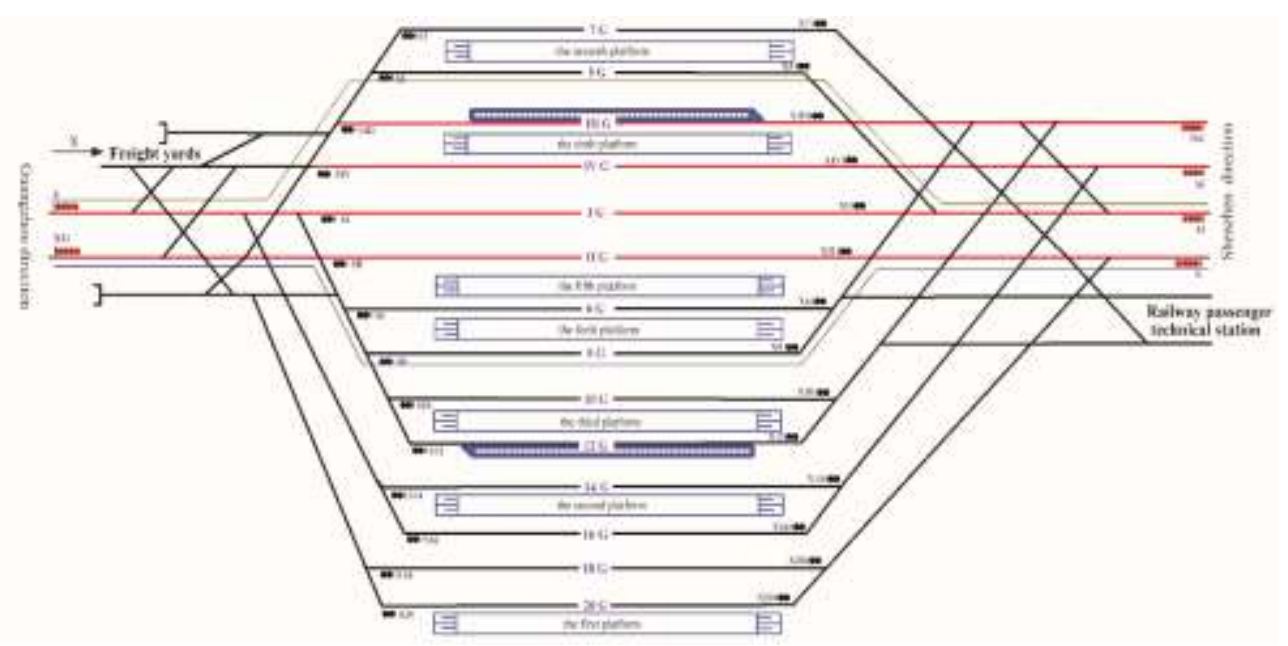

Figure 2. Schematic Layout of Guangzhou East Railway Station 


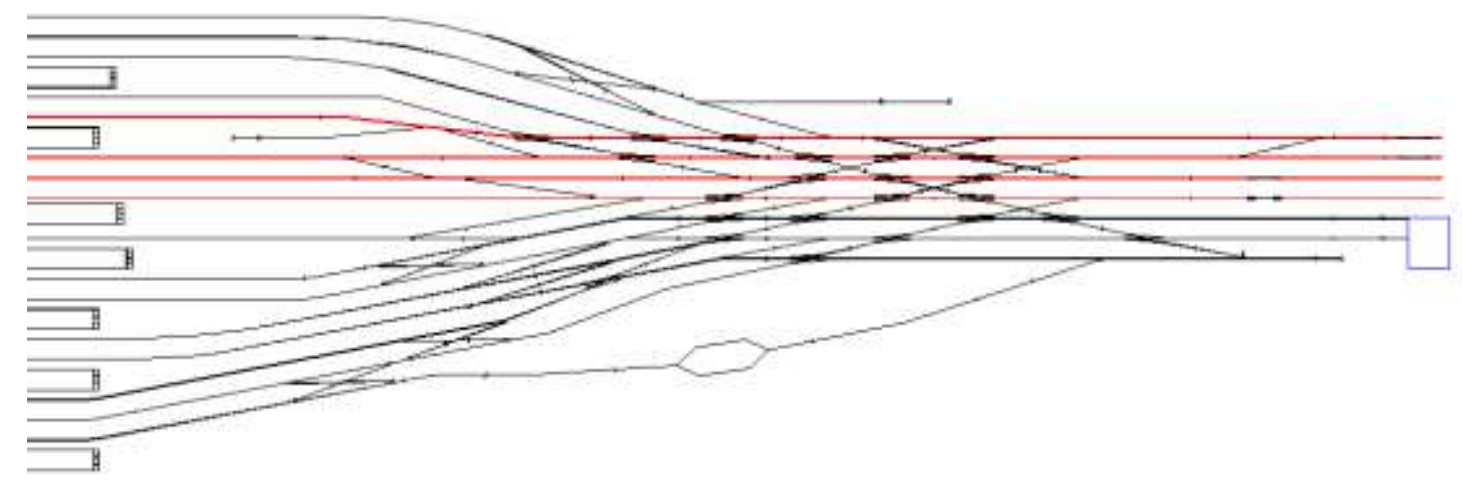

Figure 3. Interlocking Area in the Shenzhen Direction

From Figure 2, Figure 3 and the interlocking chart of the station, we can easily gain the information about all membership between tracks, platforms, routes and track-circuits. Besides, given that railway working-day (from 18:00 p.m. to 18:00 p.m. in the very next day in China), there are 264 arrival and departure trains, including 86 THPTs and 74 pairs ORPTs and TEPTs and 15 pairs arrival turnaround trains and departure turnaround trains according to its given timetable and carriages turnover plan at the Guangzhou East Railway Station.

\subsection{Results, Comparisons and Analysis}

\subsubsection{Solutions and Computing Times Comparison due to Dispatching Rules}

Considering setting 1000 iterations as the termination condition in the proposed rulebased meta-heuristic, we can acquire objective values and their computing times for gaining these good quality solutions generated from RS model under the RS interlocking formulation based on different dispatching rules, as shown in table 4.

Table 4. Results Generated from the RS model with Dispatching Rules

\begin{tabular}{|c|c|c|c|c|c|}
\hline \multirow[t]{2}{*}{ No. } & \multirow[b]{2}{*}{$\begin{array}{l}\text { Dispatching } \\
\text { rules }\end{array}$} & \multicolumn{2}{|c|}{ Initial solutions } & \multicolumn{2}{|c|}{ Good quality solutions } \\
\hline & & $\begin{array}{c}\text { Objective } \\
\text { values }\end{array}$ & $\begin{array}{l}\text { Computing } \\
\text { times }(\mathrm{ms})\end{array}$ & $\begin{array}{c}\text { Objective } \\
\text { values }\end{array}$ & $\begin{array}{l}\text { Computing } \\
\text { times }(\mathrm{ms})\end{array}$ \\
\hline 1 & EDD & 351 & 4851 & 225.6 & 1494738 \\
\hline 2 & FIFO & 413.4 & 3419 & 195.6 & 1423800 \\
\hline 3 & SPT & 283.8 & 3735 & 165 & 1481395 \\
\hline 4 & WSPT & 283.8 & 4289 & 202.2 & 1501814 \\
\hline 5 & WDSPT & 402.6 & 3445 & 322.2 & 1398792 \\
\hline 6 & BW & 427.8 & 3603 & 213.6 & 1415737 \\
\hline 7 & SL & 357 & 4152 & 193.8 & 1470734 \\
\hline 8 & FIFO-EDD & 443.4 & 3365 & 226.8 & 1473183 \\
\hline 9 & EDD-FIFO & 424.2 & 3610 & 238.8 & 1531219 \\
\hline 10 & $\begin{array}{l}\text { WSPT- } \\
\text { FIFO-EDD }\end{array}$ & 302.4 & 4439 & 159 & 1464113 \\
\hline 11 & $\begin{array}{l}\text { BW-SL- } \\
\text { FIFO-EDD }\end{array}$ & 537.6 & 4239 & 362.4 & 1466356 \\
\hline 12 & $\begin{array}{l}\text { SL-BW- } \\
\text { FIFO-EDD }\end{array}$ & 551.4 & 3786 & 225 & 1413367 \\
\hline
\end{tabular}

From Table 4, it's well known that initial solutions based on dispatching rules can be acquired around $4000 \mathrm{~ms}$, while good quality solutions can be gained about 1400000 
ms. For the average objective value after 1000 iterations, differences between good quality solutions based on different dispatching rules are quite obvious. For example, as for basic dispatching rules, the best objective value is 165 with the computing time of 1 $481395 \mathrm{~ms}$, which is gained by the rule 3 (SPT), while the worst solution (322.2 with the computing time of $1398792 \mathrm{~ms}$, which is shorter than the former one) is obtained with the rule 5 (WDSPT) and its worse $95.3 \%$ than the best one; as for composite dispatching rules, the objective value is 159 with the rule 10 (WSPT-FIFO-EDD), while the objective value is 238.8 with the rule 9 (EDD-FIFO), and obviously, the former one is much better than the latter one. Also, there are similar situation for ${ }^{\theta}$ dispatching rules. Moreover, there are much bigger differences between different kinds of dispatching rules. Similarly, there are also quite obvious differences about objective values and computing times among different dispatching rules for the initial solution generated from the RS model. Therefore, influence of dispatching rules on good quality solutions needs to be analyzed in busy complex passenger station within limited computation time.

\subsubsection{Good Quality Solutions Comparisons between RR Model and RS Model}

Similarly, considering setting another 1000 iterations as the termination condition in the proposed rule-based meta-heuristic, we can gain objective values and their computing times for gaining these good quality solutions generated from RS model and RR model based on different dispatching rules, as shown in table 5 .

Table 5. Calculation Results Gained by RR and RS model

\begin{tabular}{|c|c|c|c|c|c|}
\hline \multirow[t]{2}{*}{ No. } & \multirow[b]{2}{*}{$\begin{array}{l}\text { Dispatching } \\
\text { rules }\end{array}$} & \multicolumn{2}{|c|}{ RR model } & \multicolumn{2}{|c|}{ RS model } \\
\hline & & $\begin{array}{c}\text { Objective } \\
\text { values }\end{array}$ & $\begin{array}{l}\text { Computing } \\
\text { times }(\mathrm{ms})\end{array}$ & $\begin{array}{c}\text { Objective } \\
\text { values }\end{array}$ & $\begin{array}{c}\text { Computing } \\
\text { times }(\mathrm{ms})\end{array}$ \\
\hline 1 & EDD & 178.2 & 14067440 & 155.4 & 14193956 \\
\hline 2 & FIFO & 217.8 & 13741347 & 178.2 & 12819577 \\
\hline 3 & SPT & 186 & 14036958 & 171 & 13560807 \\
\hline 4 & WSPT & 268.8 & 16991531 & 252.6 & 19149329 \\
\hline 5 & WDSPT & 287.4 & 14279592 & 231 & 14555395 \\
\hline 6 & BW & 193.8 & 15577638 & 180.6 & 15089492 \\
\hline 7 & SL & 205.2 & 19244852 & 176.4 & 14454743 \\
\hline 8 & FIFO-EDD & 184.8 & 15253284 & 177 & 15085447 \\
\hline 9 & EDD-FIFO & 208.8 & 14676326 & 155.4 & 12914191 \\
\hline 10 & $\begin{array}{l}\text { WSPT- } \\
\text { FIFO-EDD }\end{array}$ & 195 & 15266585 & 181.8 & 12763696 \\
\hline 11 & $\begin{array}{l}\text { BW-SL- } \\
\text { FIFO-EDD }\end{array}$ & 249.6 & 12904005 & 234.6 & 13430087 \\
\hline 12 & $\begin{array}{l}\text { SL-BW- } \\
\text { FIFO-EDD }\end{array}$ & 279.6 & 15925934 & 258 & 16521307 \\
\hline - & Average & 221.25 & 15163791 & 196 & 14544836 \\
\hline
\end{tabular}

From Table 5, it's also well known that good quality solutions (weighted number of delayed trains) generated from RS model under the RS interlocking formulation are statistically better than that gained by RR model under the RR interlocking formation when the track changing strategy is adopted in such busy complex passenger station. For example, the good quality solutions of all dispatching rule in the RR model is 221.25 with the average computing time $15163791 \mathrm{~ms}$, while the good quality solutions of all dispatching rule in the RS model is 196 with the average computing time $14544836 \mathrm{~ms}$. Furthermore, the average objective value of the RS model is $11.53 \%$ better than that of the RR model, and the average computing time of the RS model is $4.08 \%$ better than that of the RR model at the same time. Hence, the good quality solution of the RS model is 
better than that of the RR model (c.f. Figure 4). Therefore, we can get the conclusion that there are different influences of different railway interlocking formations on TPPs in railway stations, especially in those busy complex rail passenger stations. We can also get the conclusion that better solutions of TPPs can be gain by RS model under the RS interlocking formulation after certain computing time when the track changing strategy is used in complex stations with partially coincident and interconnected routes. And in our opinion, that may be the main reason why the RS interlocking formulation will be applied into rail industry in many countries, especially in China's rail passenger stations. Furthermore, Figure 5 shows the difference about solution convergent speeds calculated by the RS and RR models with corresponding interlocking formulations.

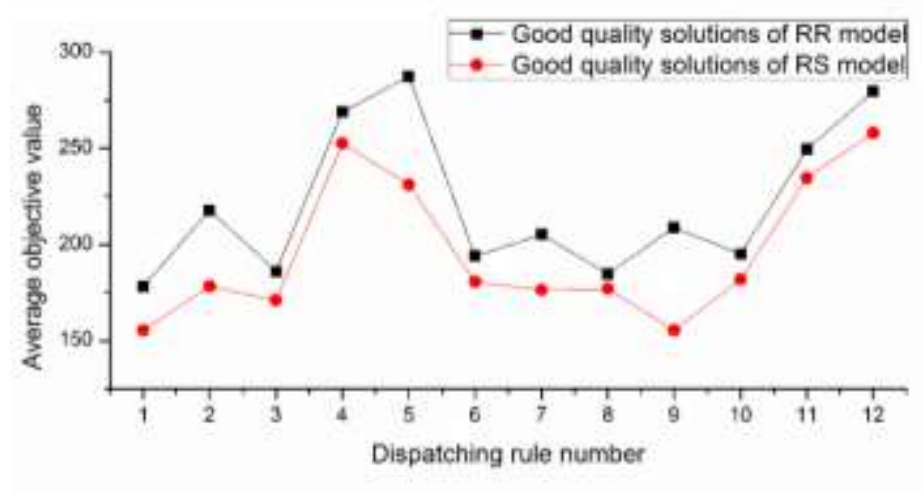

Figure 4. Good Quality Solutions Comparisons between RR and RS

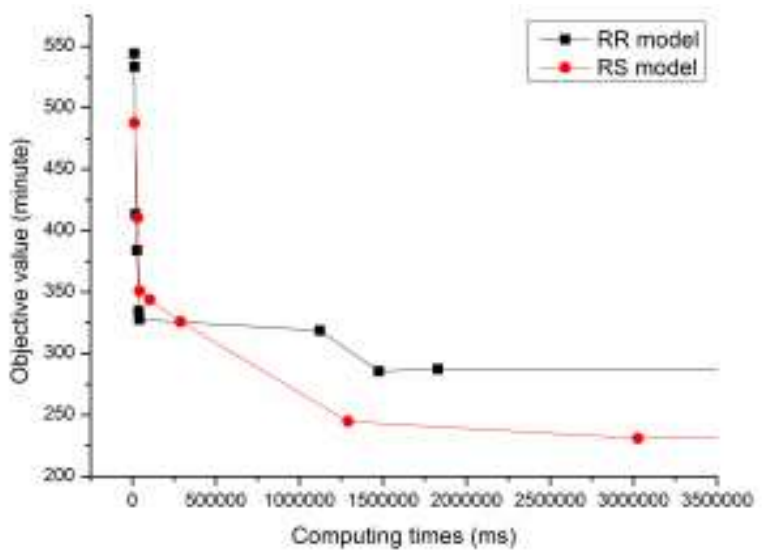

Figure 5. Solution Convergent Speed between RR and RS models

In Figure 5, It's also well known that the best-so-far solution under the RS interlocking formulation is better than the best-so-far solution of RR interlocking formulation with the dispatching rule 4 (WSPT) when the computing times are longer than $500000 \mathrm{~ms}$ (about 6 minutes). Thus, when the computing time as the termination condition is longer than 6 minutes, we'd better choose the RS model to acquire better good quality solutions.

\section{Conclusions}

In this paper, RR interlocking and RS interlocking formulations for TPP in busy complex passenger stations are compared. Firstly, we explicitly analyzed different operations of different train types conducted in complex passenger stations and proposed a microscopic simulation of train movements in the interlocking area, which is necessary 
for track-circuited formulation of TPP specific to complex stations. Then, we built job shop scheduling models with the minimum weighted number of delayed trains as their objective functions. In addition, we designed dispatching rules by incorporating classic scheduling theory into experience of dispatchers and proposed a rule-based meta-heuristic to search good quality solutions within limited computation time. Finally, we compared good quality solutions between RS and RR models with different dispatching rules, when adopting the track changing strategy in busy complex passenger stations where routes are partially coincident and interconnected. The result shows that good quality solutions generating from RS model are generally statistically better than these from RR model after certain computing time when the track changing strategy is used in complex stations. As for our future research tasks, we will explore the best dispatching rules on basis of timetable robustness and build robustness functions based on track-circuits conflict-free.

\section{Acknowledgments}

The work described in this paper is support by grants from National Natural Science Foundation of China (71501190, 71371193) and Fundamental Research Funds for the Central Universities of Central South University (2013zzts212). The authors also wish to thank the editors and reviewers for their great comments and suggestions.

\section{References}

[1] R.M. Lusby, J. Larsen, M. Ehrgott and D. Ryan, "Railway Track Allocation: Models and Methods", Operations Research Spectrum, vol. 33, no. 4, (2011), pp. 843-883.

[2] P. Sels, P. Vansteenwegen, T. Dewilde, D. Cattrysse, B. Waquet and A. Joubert, "The Train Platforming Problem: the Infrastructure Management Company Perspective". Transportation Research Part B, vol.61, no.1, (2014), pp. 55-72.

[3] V. Cacchiani, L. Galli and P. Toth, "A Tutorial on Non-periodic Train Timetabling and Platforming Problems", European Journal on Transportation \& Logistics, vol. 4, no. 3, (2014), pp. 1-36.

[4] B. Szpigel, "Optimal Train Scheduling on a Single Track Railway", Operational Research, vol. 72, (1973), pp. 344-351.

[5] A. Mascis and D. Pacciarelli, "Job-shop Scheduling with Blocking and No-wait Constrains", European Journal of Operational Research, vol. 143, no. 3, (2002), pp. 498-517.

[6] A. D'Ariano, D. Pacciarelli and M. Pranzo, "A Branch and Bound Algorithm for Scheduling Trains in a Railway Network", European Journal of Operational Research, vol. 183, no. 2, (2007), pp. 643-657.

[7] A. D'Ariano and M. Pranzo, “An Advanced Real-time Train Dispatching System for Minimizing the Propagation of Delays in a Dispatching Area under Severe Disturbances", Network and Spatial Economics, vol. 9, no. 1, (2008), pp. 63-84.

[8] C. Mannino and A. Mascis, "Optimal Real-time Traffic Control in Metro Stations", Operations Research, vol. 57, no. 4, (2009), pp. 1026-1039.

[9] F. Corman, A. D’Ariano, D. Pacciarelli and M. Pranzo, “A Tabu Search Algorithm for Rerouting Trains during Rail Operations”, Transportation Research Part B, vol. 44, no. 1, (2010), pp. 175-192.

[10] J. T. Krasemann, "Design of an Effective Algorithm for Fast Response to the Re-scheduling of Railway Traffic during Disturbances", Transportation Research Part C, vol. 20, no. 1, (2012), pp. 62-78.

[11] G. Caimi, M. Fuchsberger, M. Laumanns and M. Lüthi, "A Model Predictive Control Approach for Descrete-time Rescheduling in Busy Complex Central Railway Station Approach", Computers \& Operations Research, vol. 39, no. 11, (2012), pp. 145-155.

[12] R. M. Lusby, J. Larsen, M. Ehrgott and D. M. Ryan, "A set Packing Inspired Method for Real-time Junction Train Routing”, Computers \& Operations Research, vol. 40, no. 3, (2013), pp. 713-724.

[13] F. Corman, R. Goverde and A. D'Ariano, "Rescheduling Dense Traffic Over Busy Complex Station Interlocking Areas", Edited R. Ahuja, R. Möhring and C. Zaroliagis, Springer Berlin/Heidelberg, Berlin, vol. 5868, (2009), pp. 369-386.

[14] P. Pellegrini, G. Marlière and J. Rodriguez, "Optimal Train Routing and Scheduling for Managing Traffic Perturbations in Busy Complex Junctions", Transportation Research Part B, vol. 59, no. 1, (2014), pp. 58-80.

[15] J. Törnquist and J. A. Persson, "N-tracked Railway Traffic Re-scheduling During Disturbances", Transportation Research Part B, vol. 41, no. 3, (2007), pp. 342-362.

[16] M. Carey and S. Craville, "Scheduling and Platforming Trains at Busy Complex Stations", Transportation Research Part A, vol. 37, no. 3, (2003), pp. 195-224.

[17] M. Carey and I. Crawford, "Scheduling Trains on a Network of Busy Complex Stations", Transportation Research Part B, vol. 41, no. 2, (2007), pp. 159-178. 
[18] L. G. Kroon, H. E. Romeijn and P. J. Zwaneveld, "Routing Trains through Railway Stations: Busy Complexity Issues", European Journal of Operational Research, vol. 98, no. 3, (1997), pp. 485-498.

[19] M. Samà, C. Meloni, A. D'Ariano and F. Corman, "A Multi-criteria Decision Support Methodology for Real-time Train Scheduling”, Journal of Rail Transport Planning \& Management, vol. 5, no. 3, (2015), pp. 146-162.

[20] W. Fang, S. Yang and X. Yao, "A Survey on Problem Models and Solution Approaches to Rescheduling in Railway Networks", IEEE Transactions on Intelligent Transportation Systems, vol. 16, no.6, (2015), pp. 2997-3016.

[21] Q. Lu, M. Dessouky and R. C. Leachman, "Modeling Train Movements through Busy Complex Rail Networks", Acm Transactions on Modeling \& Computer Simulation, vol. 14, no. 1, (2004), pp. 48-75.

[22] J. Rodriguez, "A Constraint Programming Model for Real-time Train Scheduling at Junctions", Transportation Research Part B, vol. 41, no. 2, (2007), pp. 231-245.

[23] P. J. Zwaneveld, L. G. Kroon, H. E. Romeijn, M. Salomon, S. D. Peres, S. P. M. V. Hoesel and H. W. Ambergen, "Routing Trains through Railway Stations: Model Formulation and Algorithms", Transportation Science, vol. 30, no. 3, (1996), pp. 181-194.

[24] R. L. Graham, E. L Lawler, J. K. Lenstra and A. R. Kan, "Optimization and Approximation in Deterministic Sequencing and Scheduling: a Survey”, Annals of discrete mathematics, vol. 5, (1979), pp. 287-326.

[25] Y. Zhang, D. Lei, M. Wang and Q. Zeng, "Dispatching Rules: Track Utilization Scheduling Problem in Railway Passenger Stations”, ICTIS 2013, Wuhan, China, (2013), June 01-02.

[26] E.L. Lawer and J.M. Moore, "A Function Equation and its Application to Resource Allocation and Sequencing Problems", Management Science, vol. 16, no. 1, (1969), pp. 77-84.

[27] R. Garey and D. S. Johnson, "Two-processor Scheduling with Start-times and Deadlines", SIAM Journal on Computing, vol. 6, no. 3, (1977), pp. 416-426.

[28] M. Pindeo, "Scheduling: Theory, Algorithms and Systems", Prentice Hall, USA, (2002).

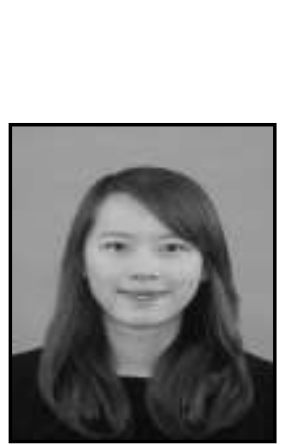

\section{Authors}

Qiongfang Zeng, he is a $\mathrm{PhD}$ candidate at the School of Traffic and Transportation Engineering, Central South University, China. Her research is focus on train platforming problem and urban public transport integration problem. Besides, she is also interested in modern optimization theories such as modern scheduling theory and good at building mathematic modes based on these theories.

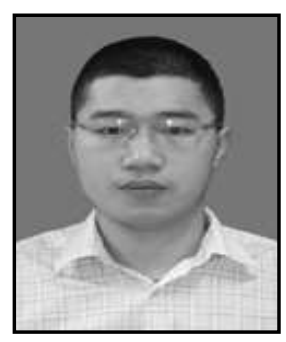

Yinggui Zhang, he is a $\mathrm{PhD}$ graduated from Central South University, major in traffic and transportation planning and management. His research interest is in the area of rail and road transportation management, and specializes in transportation scheduling model and algorithm design. He is currently working at the School of Traffic and Transportation Engineering, Central South University as a Lecturer and Master Supervisor in China.

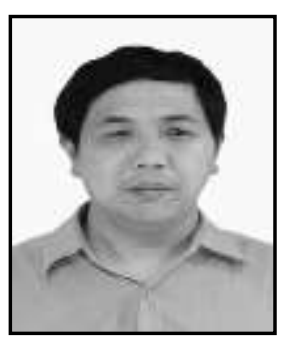

Dingyou Lei, he has over two decades of experience in the field of traffic and transportation planning and management. He specializes in traffic and transportation model and information management. He is a famous expert in rail transport safety. He is currently working at the School of Traffic and Transportation Engineering, Central South University, as a professor and the head of Traffic and Transportation System Institute. 


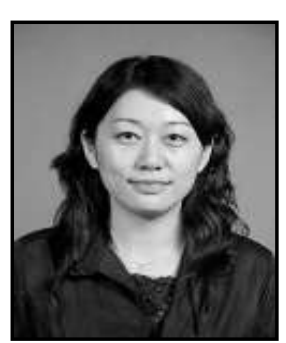

Li Wang, she is a $\mathrm{PhD}$ graduated from Beijing Jiaotong University, major in traffic and transportation Engineering. Her research interest is in the area of rail transportation and safety management, and specializes in the active safety protection technology of rail transport system. She is currently working at the School of Traffic and Transportation, Beijing Jiaotong University as a Lecturer in China. 